12

\title{
Динамика катодных пятен в аксиальном магнитном поле на спаде импульса тока в сильноточной вакуумной дуге
}

\author{
(C) А.А. Логачев ${ }^{1}$, С.В. Клочко ${ }^{2}$, И.Н. Полуянова ${ }^{2}$ \\ ${ }^{1}$ Физико-технический институт им. А.Ф. Иоффе РАН, Санкт-Петербург \\ 2 ПГ „Таврида Электрик“, Москва \\ E-mail: logatchev@mail.ioffe.ru
}

Поступило в Редакцию 23 июня 2017 г.

Исследована катодная привязка сильноточной вакуумной дуги на спаде импульса тока. Использовались контакты, генерирующие аксиальное магнитное поле. Дано количественное объяснение обнаруженного изменения формы привязки вблизи нуля тока, которое показало удовлетворительное согласие с результатами эксперимента.

DOI: 10.21883/PJTF.2017.20.45150.16932

Интерес к исследованию сильноточного вакуумного дугового разряда вызван широким использованием вакуумной коммутационной аппаратуры, прежде всего в высоковольтных сетях переменного тока с частотой $50-60 \mathrm{~Hz}[1,2]$. Важнейшим направлением работы является обеспечение максимальной отключающей способности вакуумных дугогасительных камер (ВДК) при заданных габаритах контактной системы (КС). Чтобы достичь этого, необходимо обеспечить однородное распределение тепловой нагрузки по поверхности контактов. Одним из наиболее распространенных способов оптимизации разряда является использование специальных конструкций КС, которые генерируют аксиальное магнитное поле (АМП) [3]. Поэтому исследования сильноточных вакуумных дуг, питаемых синусоидальными импульсами тока и находящихся под действием АМП, ведутся интенсивно уже многие годы. Отметим, что АМП, генерируемое самими КС, существенно неоднородно и отстает по фазе от тока. Это следствие того, что в таких КС протекающий (коммутируемый) ток используется для генерации АМП. 
В исследованиях сильноточных вакуумных дуг, находящихся под действием АМП, традиционно изучается развитие дуги после ее поджига по мере нарастания тока. Наибольший интерес обычно сосредоточен на состоянии дуги вблизи максимума тока. Однако успешное срабатывание ВДК определяется состоянием контактов вблизи нуля тока. Исследования процессов в дуге и на контактах на спаде тока и, особенно, вблизи нуля тока немногочисленны. Их развитие весьма актуально (см. работу [4] и ссылки в ней).

Недавно в [5] при исследовании дуговых процессов при спаде тока в КС, генерирующих АМП, было обнаружено, что вблизи нуля тока катодные пятна (КП), локализованные вблизи оси КС, отмирают раньше, чем на периферии контактов. Предложено качественное объяснение этого эффекта, основанное на упомянутом выше сдвиге фаз АМП и дугового тока, протекающего через КС. Сдвиг фаз вызван вихревыми токами, генерирующимися в КС при уменьшении дугового тока. Обнаруженное явление не только имеет физический интерес, но также важно для разработки и модернизации ВДК для выключателей переменного и постоянного тока. Однако в [5] приведены лишь качественные результаты эксперимента, объяснение которых тоже сугубо качественное.

Нами также было исследовано это явление. Получены количественные результаты, характеризующие его, и предложено их объяснение, позволяющее получить с помощью несложного численного расчета количественные характеристики. Сравнение результатов эксперимента и расчета дало удовлетворительный результат.

Эксперименты проводились в разборной вакуумной камере при непрерывной откачке (давление $\sim 10^{-4} \mathrm{~Pa}$ ). Средняя скорость движения контакта $\sim 1 \mathrm{~m} / \mathrm{s}$, финальный зазор $h \sim 6-7 \mathrm{~mm}$. Использовались стандартные промышленные КС диаметром $D=64 \mathrm{~mm}(h / D \ll 1)$ с накладкой из медь-хромовой композиции, генерирующие АМП. Диаметр плоской части контакта $D_{0}=51 \mathrm{~mm}$. Разряд питался импульсами тока амплитудой до $60 \mathrm{kA}$, по форме близкими к полуволне синусоиды $50 \mathrm{~Hz}$. Синхронизация обеспечивала разрыв контактов в пределах первой миллисекунды после включения тока.

Съемка производилась сбоку под углом примерно $6^{\circ}$ к поверхности катода. Использовалась высокоскоростная видеокамера Phantom MIRO M310 с макрообъективом Carl Zeiss 100/2. Производительность камеры составляет $3.2 \mathrm{Gpx} / \mathrm{s}$, что при размерах кадра $512 \times 144$ рх обеспечивает

Письма в ЖТФ, 2017, том 43, вып. 20 


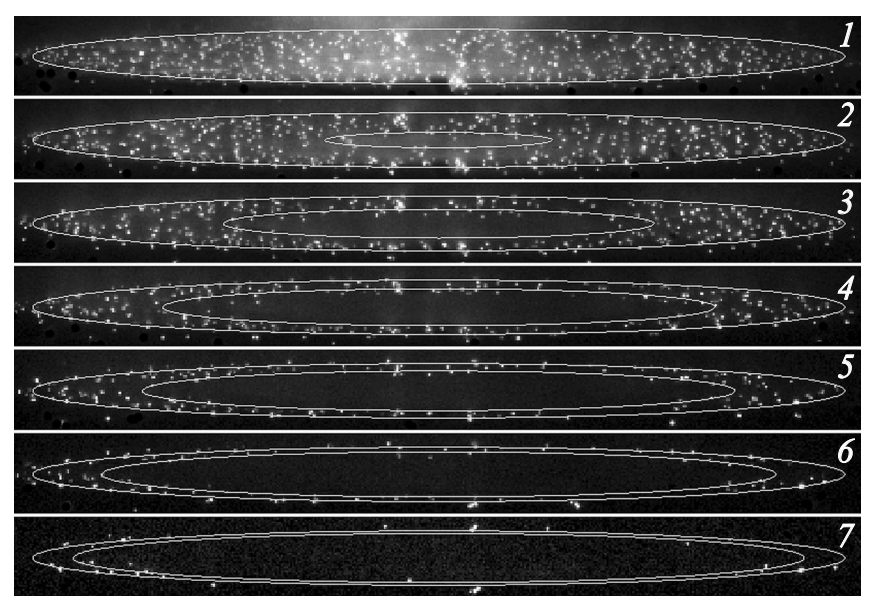

Рис. 1. Выборка кадров видеосъемки катода. Полуволна синусоидального тока $10.2 \mathrm{~ms}$, амплитуда $55.5 \mathrm{kA}$. Экспозиция $10 \mu \mathrm{s}$. 1 - время от фронта тока $t=8.2 \mathrm{~ms}$, мгновенное значение тока $I=20.1 \mathrm{kA} ; 2-t=8.5 \mathrm{~ms}, I=15.4 \mathrm{kA}$; $3-t=8.8 \mathrm{~ms}, I=11.3 \mathrm{kA} ; 4-t=9.1 \mathrm{~ms}, I=7.9 \mathrm{kA} ; 5-t=9.4 \mathrm{~ms}$, $I=5.1 \mathrm{kA} ; 6-t=9.7 \mathrm{~ms}, I=2.7 \mathrm{kA} ; 7-t=10 \mathrm{~ms}, I=0.5 \mathrm{kA}$. Эллипсами обозначены край плоской части контакта и внутренняя граница области, занятой КП.

съемку $40000 \mathrm{fps}$ (интервал между кадрами $25 \mu \mathrm{s}$ ). Использовался красный светофильтр КС-18 и нейтральные фильтры.

Анализ результатов видеосъемки поверхности катода показал, что развитие разряда при токах $I \geq 30 \mathrm{kA}$ завершается через 4-5 ms после поджига. К этому времени катодная привязка разряда вполне однородно распределяется по всей площади плоской части контакта. На спаде тока, первые несколько миллисекунд после максимума тока, однородность привязки и ее размер сохраняются, хотя средняя плотность тока очевидным образом уменьшается. Общее количество КП изменяется пропорционально току. Однако примерно за $1 \mathrm{~ms}$ до нуля тока становится видно, что форма привязки меняется. КП исчезают из приосевой области, форма привязки становится кольцевой. Вместо отмирающих в приосевой области КП образуются новые КП на периферии. КП образуются в результате взрывоэмиссионных процессов 


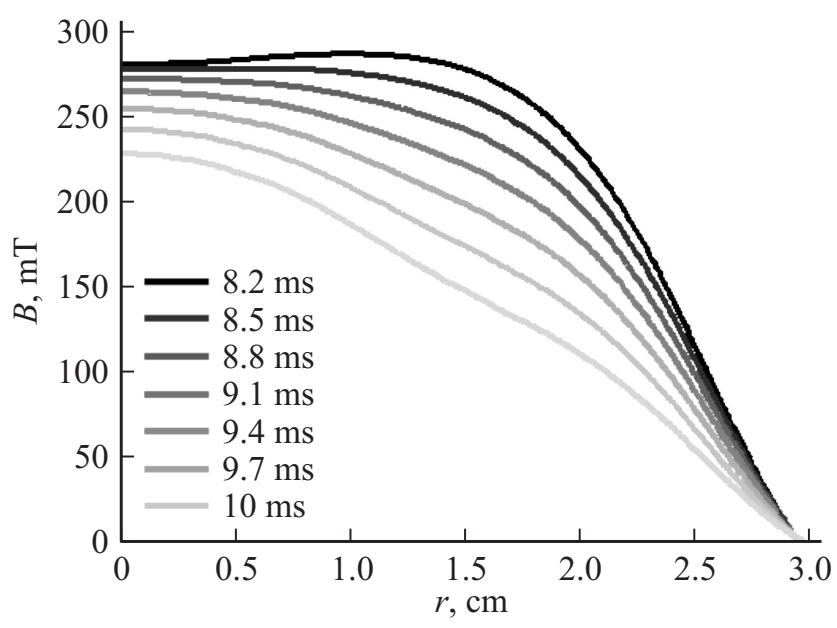

Рис. 2. Радиальные зависимости индукции аксиального магнитного поля.

на микровыступах, образовавшихся при выбросе металла из кратера КП, горевшего в предшествующие моменты времени [6]. Внешний радиус кольца $r_{1}$ примерно равен радиусу плоской части контакта $\left(r_{1}=D_{0} / 2 \approx 25 \mathrm{~mm}\right)$. Внутренний радиус кольца $r_{2}$ с течением времени быстро увеличивается, и непосредственно перед нулем тока небольшое количество оставшихся КП располагается на самом краю плоской части контакта. В качестве примера на рис. 1 приведена выборка кадров из результатов съемки импульса тока амплитудой $55.5 \mathrm{kA}$ (разрыв контактов - поджиг дуги - произошел через $0.4 \mathrm{~ms}$ после включения тока). Описанное выше изменение формы привязки хорошо видно из видеосъемки.

Объяснение наблюдаемого явления, как видно из дальнейшего рассмотрения, требует знания динамики АМП внутри промежутка при спаде и занулении тока. На рис. 2 представлены рассчитанные в среде COMSOL радиальные зависимости индукции AMП $B(r)$ в моменты времени, соответствующие кадрам (рис. 1). Сравнение рис. 1 и 2 показывает, что КП вытесняются в область меньшего АМП.

Коротко перечислим полученные ранее результаты [7-9], которые позволяют объяснить описанное выше изменение формы катодной

Письма в ЖТФ, 2017, том 43, вып. 20 
привязки вблизи нуля тока. В [7] было исследовано воздействие однородного внешнего АМП на короткую $(h / D \ll 1)$ сильноточную вакуумную дугу и показано, что состояние дуги определяется приэлектродными процессами, прежде всего динамикой КП под действием результирующего магнитного поля - собственного поля дугового тока и внешнего однородного АМП. Было обнаружено, что в дуге при заданном токе $I$ можно определить два характерных значения индукции АМП: $B_{1}$ и $B_{2}$. В диапазоне $B_{1}<B<B_{2}$ КП занимают всю торцевую поверхность контакта, при этом напряжение на дуге минимально и практически не зависит от $B$. Средняя плотность тока при этом составляет $j_{0}=4 I /\left(\pi D_{0}^{2}\right)$. При $B<B_{1}$ часть КП выходит на боковую поверхность, напряжение повышенное. Средняя плотность тока при этом уменьшается: $j_{1}<j_{0}$. При $B>B_{2}$ область, занятая КП, сокращается; напряжение растет при увеличении $B$. Отметим, что разным значениям тока соответствуют разные величины $B_{1}$ и $B_{2}$ (бо́льшим токам отвечают бо́льшие поля). Также было обнаружено, что при $B>B_{2}$ средняя плотность тока в привязке зависит только от $B$ (растет с ростом $B$ ) и не зависит от тока дуги при изменении его в пределах от 3 до $11.5 \mathrm{kA}$. Эта плотность тока была названа нормальной $\left(j_{\text {norm }}\right)$ [7]. Очевидно, что $j_{\text {norm }} \geq j_{0}$.

Продолжение работы показало, что обнаруженная закономерность сохраняется при изменении тока дуги в значительно более широких пределах (на порядок величины и более) [8]. Измерения в $[7,8]$ проводились в дугах длиной $h=6 \mathrm{~mm}$ с медь-хромовыми электродами. Аппроксимация экспериментальных результатов $[7,8]$ позволила получить выражения для $B_{1}\left(j_{0}\right)$ [3] и для $j_{\text {norm }}(B)$, в которых $j$ измеряется в $\mathrm{kA} / \mathrm{cm}^{2}$, а $B-$ в теслах:

$$
\begin{gathered}
B_{1}=0.086 j_{0}, \\
j_{\text {norm }}(B)=2.2 B^{0.42} .
\end{gathered}
$$

Структура катодной привязки в неоднородных АМП была исследована в работе [9]. Было обнаружено, что формирующие привязку КП располагаются так, чтобы в соответствии с принципом Штеенбека напряжение дуги было минимальным, т. е. занимают часть поверхности катода, над которой выполнено условие $B_{1}<B<B_{2}$. В частности, в сильных полях колоколообразной формы КП перераспределялись на периферию, образуя привязку кольцевой формы.

Письма в ЖТФ, 2017, том 43, вып. 20 


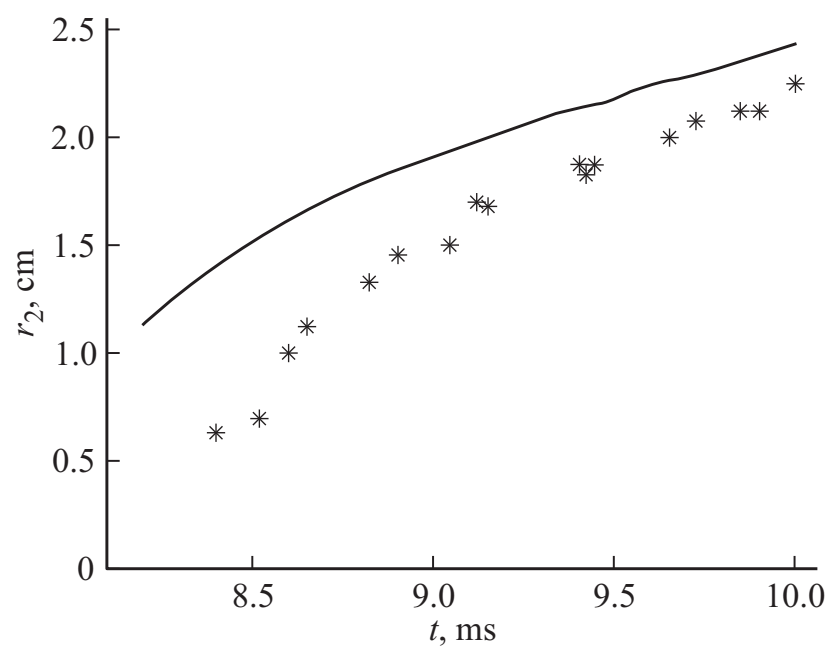

Рис. 3. Положение рассчитанной (сплошная линия) и оцененной из результатов видеосъемки (звездочки) внутренней границы области, занятой КП.

Исходя из этих результатов мы предполагаем, что в КС, генерирующих АМП, перераспределение КП объясняется тем, что на спаде тока индукция АМП в приосевой области спадает медленнее, чем на периферии (рис. 2), скорость ее уменьшения значительно меньше, чем скорость уменьшения тока. Вследствие этого в приосевой области индукция АМП превышает значение $B_{2}$, соответствующее мгновенному значению тока. Поэтому КП перераспределяются на периферию катода, над которой индукция АМП ниже, чем над приосевой областью, причем на ту часть поверхности, над которой $B_{1}<B<B_{2}$, так как такой структуре привязки соответствует минимальное напряжение на дуге.

Для выбранного нами для иллюстрации режима $(I=55.5 \mathrm{kA})$ определим значения $r_{1}$ и $r_{2}$ в различные моменты времени, которым соответствуют различные мгновенные значения тока. Внешний радиус привязки $r_{1}$ определим, сопоставляя расчеты по формуле (1) с расчетами АМП (выборка из этих расчетов приведена на рис. 2). Полученные значения $r_{1}$ оказываются на $1-3 \mathrm{~mm}$ больше радиуса плоской части контакта $D_{0} / 2$. КП практически не выходят за границу плоской части („эффект края““ [3]); таким образом, величина $r_{1}$ примерно равна $D_{0} / 2$.

Письма в ЖТФ, 2017, том 43, вып. 20 
Определив $r_{1}$, можно найти внутренний радиус $r_{2}$. При этом мы исходим из того, что $j_{\text {norm }}\left(B_{2}\right) \approx j$. Средняя плотность тока в привязке $j=I /\left(\pi\left(r_{1}^{2}-r_{2}^{2}\right)\right)$. Тогда значение $r_{2}$ можно получить путем численного решения уравнения (2), которое при $B=B_{2}$ может быть записано в виде $2.2 B^{0.42} \approx I /\left(\pi\left(r_{1}^{2}-r_{2}^{2}\right)\right)$.

Сравнение результатов расчета внутренней границы катодной привязки с оценками ее размера, сделанными на основе результатов видеосъемки дуги, представлены на рис. 3. Согласие можно считать удовлетворительным, несмотря на использование в расчете среднего значения плотности тока.

Можно заключить, что полученные ранее зависимости характеристик катодной привязки сильноточной вакуумной дуги от индукции однородного внешнего АМП, дополненные принципом минимума Штеенбека, позволяют как качественно, так и количественно описать форму катодной привязки в неоднородном поле вблизи нуля тока в используемых на практике КС, генерирующих АМП.

Авторы признательны С.М. Школьнику за обсуждения и В.В. Яковлеву за помощь в численных расчетах.

\section{Список литературы}

[1] Slade P.G. The vacuum interrupter: Theory, design and application. CRC Press, 2008. $528 \mathrm{p}$.

[2] Jia S., Li X., Shi Z., Wang L. // IEICE Trans. Electron. 2012. V. E95-C. N 9. P. $1452-1458$.

[3] Chaly A.M. // IEEE Trans. Plasma Sci. 2005. V. 33. N 5. P. 1497-1503.

[4] Chaly A.M., Poluyanova I.N., Yakovlev V.V. et al. // 27th ISDEIV. China, 2016. V. 1. P. $302-305$.

[5] Song X., Shi Z., Jia S., Wang L., Zhang Y. // 25th ISDEIV. Russia, 2012. V. 1. P. 289-292.

[6] Гашков М.А., Зубарев Н.М., Месяи, Г.А., Уйманов И.В. // Письма в ЖТФ. 2016. T. 42. B. 16. C. $48-55$.

[7] Chaly A.M., Logatchev A.A., Shkolnik S.M. // IEEE Trans. Plasma Sci. 1999. V. 27. P. 927

[8] Chaly A., Logachev A., Shkolnik S., Zabello K. // 19th ISDEIV. China, 2000. V. 1. P. 286-289.

[9] Chaly A.M., Logatchev A.A., Zabello K.K., Shkol'nik S.M. // IEEE Trans. Plasma Sci. 2003. V. 31. N 5. P. 884-889.

Письма в ЖТФ, 2017, том 43, вып. 20 